\title{
CRISE CONVULSIVA COMO MANIFESTAÇÃO DE INSULINOMA: UM RELATO DE CASO
}

\author{
SEIZURE AS INSULINOMA APRESENTATION: A CASE REPORT
}

Ana Cristina Boni Brandão¹, Laís de Medeiros , Júlia Vieira Oberger Marques², Caio Cesar Cervi Lagana², Marcela $\mathrm{Robl}^{2}$, Debora Cristina Besen 2, Patrícia Oliboni do Amaral ${ }^{2}$, Neudir Frare Junior ${ }^{2}$, Adriane Maria Rodrigues³;

\section{RESUMO}

Introdução: 0 insulinoma é um tumor neuroendócrino com prevalência de 4:1.000.000 habitantes, sem predominância por sexo ou idade, e representa cerca de 1-2\% das neoplasias pancreáticas. Portadores da doença apresentam sintomas de hipoglicemia que são manifestados devido à hipersecreção de insulina. Objetivo: Relatar um caso de insulinoma diagnosticado após crise convulsiva. Relato de caso: Paciente feminina, 31 anos, interna por crises convulsivas causadas por hipoglicemia com início há 3 anos. Durante a internação iniciou-se investigação sendo constatado hiperinsulinemia, porém, apesar da suspeição, exames de imagem disponiveis não detectaram a presença de insulinoma. Foi submetida a intervenção cirúrgica com USG intraoperatório sem sucesso. No pós operatório, necessitou de uso de análogos de somatostatina para controle da doença e evoluiu sem presença de novos episódios de hipoglicemia. Conclusão: Insulinoma é um raro tumor que secreta insulina, levando a sinais e sintomas de hipoglicemia. A excisão cirúrgica do tumor, quando possível, leva a cura da maioria dos pacientes. Naqueles não curados pela cirurgia, o uso de análogos de somatostatina representam uma segunda opção de tratamento.

Descritores: Insulinoma; hiperinsulinismo; hipoglicemia;

\section{ABSTRACT}

Introduction: Insulinoma is a neuroendocrine tumor with an incidence of 4 per million, with no difference of prevalence by sex or age, and represents about $1-2 \%$ of the pancreatic neoplasia. This patient has hypoglycemic symptoms, manifested due to insulin hypersecretion. Objective: Report an insulinoma case. Case report: A female patient, 31 years old, admitted in 2011 due to convulsions that began three years ago, caused by hypoglycemia. In the hospital, it was found hyperinsulinism. However, the image exams were negative to insulinoma. After undergoing surgical procedure, the patient continued with hypoglycemia. Using longacting somatostatin analog, therefore, the patient had no further episodes of hypoglycemia. Conclusion: Insulinoma is a rare tumor which leads to elevated insulin levels. The surgery is favorable and leads to healing in most cases. The use of long-acting somatostatin analog is the second treatment option.

Keywords: Insulinoma; hyperinsulinism; hypoglycemia;

1- Acadêmicas do curso de Medicina da Universidade Federal do Paraná;

2- Residentes de Endocrinologia e Metabologia do Serviço de Endocrinologia e Metabologia da Universidade Federal do Paraná;

3- Médica Endocrinologista do Serviço de Endocrinologia e Metabologia da Universidade Federal do Paraná; 


\section{REVISTA MÉDICA DA UFPR}

\section{INTRODUÇÃO}

Insulinoma é um raro tumor neuroendócrino produtor de insulina com prevalência de 4/1.000.000 habitantes ${ }^{2}$. A manifestação mais comum da doença é a hipoglicemia ${ }^{1}$. Pode ser encontrado isolado ou como parte da síndrome de neoplasia endócrina múltipla tipo 1 (NEM$1)^{5}$. O diagnóstico de insulinoma é estabelecido pela alta concentração de insulina sérica durante um episódio provocado ou induzido de hipoglicemia, como no teste de 72 horas de jejum ${ }^{1}$. A excisão cirúrgica tem um grande potencial de cura nestes pacientes, com taxa de sucesso entre $77-100 \% 5,8$. USG transabdominal, tomografia computadorizada e ressonância nuclear magnética são usados para determinar a localização da lesão ${ }^{8}$.

\section{RELATO DE CASO}

Paciente C.V., feminina, 31 anos, interna por hipoglicemia manifestada por convulsões tônico-clônicas generalizadas que se iniciaram há 3 anos. Além das crises convulsivas, a paciente relatava quadros de tontura, cefaleia, taquicardia e sudorese. Estava em investigação desde o início dos sintomas com realização de exames como Ressonância Nuclear Magnética (RNM) de crânio, Tomografia Computadorizada de Crânio e realizou cinco eletroencefalogramas, todos normais. Naquele momento, a paciente estava em uso de carbamazepina, ácido valpróico e valproato de sódio sem controle adequado das crises.

Além dos exames de imagem anteriormente citados, realizou exames laboratoriais 3 meses antes da internação, nos quais foi constatado hipoglicemia e hiperinsulinemia.

$\mathrm{Na}$ internação, foi submetida à investigação da hipoglicemia que afastou causas exógenas para tal e diante da suspeita, iniciou-se a pesquisa para localização do insulinoma. No entanto, os exames de imagem, incluindo PET-SCAN, não encontraram a localização do tumor com permanência dos sintomas glicopênicos com glicemia atingindo níveis entre 7 e $33 \mathrm{mg} / \mathrm{dL}$.

Excluída a causa factícia, o Serviço de Endocrinologia e Metabologia do Hospital de Clínicas da UFPR (SEMPR) indicou investigação com ultrassonografia (USG) pancreática com biópsia por congelação e possibilidade de ressecção do tumor no mesmo tempo cirúrgico. A paciente foi submetida ao procedimento cirúrgico de enucleação do insulinoma com USG intraoperatória. No pós-operatório, no entanto, manteve quadro de hipoglicemia.

No laudo anatomopatológico, foi demonstrado neoplasia neuroendócrina característica de insulinoma e de caráter benigno, medindo 1 x 0,7 x 0,5 cm. Tendo em vista a não resolução do quadro, foi iniciada terapia com octreotide 30mg logo após a abordagem.

No acompanhamento no SEMPR, a paciente se manteve assintomática e sem novos episódios de hipoglicemia sendo mantida a terapia com o análogo da somatostatina, com plano de diminuição da dose e aumento do intervalo entre as doses para as consultas seguintes.

Nova RNM de abdome foi realizada aproximadamente 1 ano após a abordagem cirúrgica sendo constatado apenas status pós cirúrgico sem evidência de tumor residual.

Após 3 anos da intervenção cirúrgica manteve-se sem episódios de hipoglicemia. Porém, durante o acompanhamento, em nova RNM de abdome foi constatada pequena área noduliforme em pâncreas, hipercaptante do meio de contraste na fase arterial em porção cefálica, próximo à transição corpo/colo mediante ao colédoco distal com 1,2 × 1,1 x 0,7 cm, apresentando rápido wash out. Pela possibilidade de recidiva tumoral após discussão com o Serviço de Radiologia do Hospital de Clínicas da UFPR foi definido que a imagem provavelmente se tratava de cicatriz pós-cirúrgica sem lesão recidivada ou nova lesão.

Para melhor avaliação da imagem, nova RNM abdome foi solicitada e evidenciado aparente nódulo hipervascular na cabeça do pâncreas, medindo $0,6 \mathrm{~cm}$ no maior eixo sem impregnação pelo contraste de caráter indeterminado. Restante do pâncreas permanecia com sinal, dimensões e morfologias normais, sem dilatações ductais. Manteve acompanhamento com imagem e acompanhamento clínico. Não houve mudança no tamanho e características da imagem bem como manteve-se assintomática e sem novos episódios de hipoglicemia.

\section{DISCUSSÃO}

Insulinoma é um raro tumor neuroendócrino que causa hiperinsulinemia e como consequência $73 \%$ dos pacientes apresentam sintomas de hipoglicemia exclusivamente em períodos de jejum ${ }^{1}$. Sua incidência é de 4 casos por milhão ${ }^{2}$ e é a causa mais comum de hipoglicemia associada com hiperinsulinismo endógeno ${ }^{3}$. Representa cerca de 1-2\% das neoplasias pancreáticas ${ }^{3}$, podendo ocorrer em qualquer idade e sem predominância por sexo ${ }^{3}$. Esses tumores podem estar distribuídos por toda a extensão pancreática, porém mais comumente estão em cauda e corpo 3 com menos que $20 \mathrm{~mm}^{4}$. Geralmente ocorrem isolados, mas podem estar associados com neoplasia endócrina múltipla tipo 1 (NEM1) ${ }^{5}$. Dentre os insulinomas $10 \%$ são metastáticos, e pelo menos $10 \%$ são múltiplos, porém se comportam como tumores benignos ${ }^{4}$.

Em 1935, Whipple e Frantz descreveram o diagnóstico de hipoglicemia através de uma tríade: hipoglicemia provocada pelo jejum, glicose sérica < $50 \mathrm{mg} / \mathrm{dl}$ no momento dos sintomas e melhora dos sintomas após administração de glicose ${ }^{6}$. 0 diagnóstico de insulinoma é estabelecido pela alta concentração de insulina sérica durante um episódio provocado ou induzido de hipoglicemia, como no teste de 72 horas de jejum para um paciente com hipoglicemia em jejum ${ }^{1}$. 
Tabela 1: Valores de insulina, glicose e peptídeo $C$ antes e após a cirurgia de enucleação do insulinoma.

\begin{tabular}{|c|c|c|c|c|}
\hline DATA & $\begin{array}{c}\text { INSULINA } \\
(\mu \mathrm{U} / \mathrm{mL})\end{array}$ & $\begin{array}{c}\text { GLICOSE } \\
(\mathrm{mg} / \mathrm{dL})\end{array}$ & $\begin{array}{c}\text { PEPTÍDEO C } \\
(\mathrm{nmol} / \mathrm{L})\end{array}$ & $\begin{array}{c}\text { Razão insulina } \\
(\mathrm{mU} / \mathrm{ml}) / \\
g l i c o s e(\mathrm{mg} / \mathrm{dl})\end{array}$ \\
\hline Pré Operatório & 170 & 46 & 1,84 & 3,6 \\
\hline Pré Operatório & 26,4 & 31 & 1,94 & 0,8 \\
\hline Pós Operatório & 259 & 53 & - & 4,8 \\
\hline
\end{tabular}

Os sintomas causados podem ser divididos em neurológicos (neuroglicopênicos) e adrenérgicos ${ }^{7}$. Entre os sintomas neurológicos estão a diplopia, visão turva, estado mental alterado, comportamento anormal, amnesia, coma e convulsões ${ }^{7}$. Os sintomas causados pela resposta adrenérgica incluem sudorese, ansiedade, palpitações, fraqueza, tremores e náuseas ${ }^{7}$. A paciente do caso em questão apresentava quadros de convulsões recorrentes, sem a devida investigação mesmo diante da evidência clínica do quadro de hipoglicemia e em uso de diversos anticonvulsivantes sem resposta.

São critérios para diagnóstico de insulinoma: nível de glicose sérica < 50mg/dl com sintomas de hipoglicemia, melhora dos sintomas após alimentação, nível de insulina plasmática aumentada $(\geq 5 \mu \mathrm{U} / \mathrm{ml})$, aumento do nível de peptídeo $C(\geq 0,2 \mathrm{nmol} / \mathrm{l})$, nível de proinsulina aumentado ( $\geq 5 \mathrm{pmol} / \mathrm{l}$ ) e ausência de sulfonilureia no plasma5. Estes testes devem ser feitos durante a crise de hipoglicemia após o jejum prolongado5. Outros diagnósticos diferenciais devem ser excluídos como nesidioblastose, hipoglicemia induzida por sulfonilureia ou hipoglicemia autoimune, pela presença de anticorpos contra a insulina ou seu receptor ${ }^{1}$.

Uma vez que a excisão cirúrgica completa é potencialmente curativa para os insulinomas, determinar a localização da lesão é essencial ${ }^{5}$. Estudos não invasivos incluem USG transabdominal, tomografia computadorizada e ressonância nuclear magnética 5 . Porém os resultados podem ser inconclusivos, especialmente em lesões menores que $2 \mathrm{~cm}^{5}$. Neste caso, com um tumor medindo 1 x $0,7 \times 0,5 \mathrm{~cm}$, a cirurgia não obteve o sucesso esperado e crises de hipoglicemia ocorreram após o procedimento. Questiona-se neste momento se o tumor foi totalmente retirado ou se a falha foi devido ao tamanho da lesão. Exames de imagem desta paciente após a cirurgia demonstraram apenas imagens cicatriciais e nenhum foco provável de ressecção incompleta. 0 manejo cirúrgico em pacientes com o diagnóstico bioquímico de insulinoma é favorável, com uma taxa de sucesso entre $77-100 \%{ }^{8}$. USG intra operatório e palpação podem ser realizados para localizar efetivamente o tumor e guiar na dissecção ${ }^{8}$. Após a excisão cirúrgica do insulinoma, a maioria dos pacientes obtém taxas de cura aproximadas a 80-96\% ${ }^{9}$.

Desde que a presença de receptores de somatostatina foi observada em insulinomas, terapêutica com análogos de somatostatina, como o octreotide, pode ser oferecida como nova opção aos pacientes que apresentam falha no tratamento cirúrgico ${ }^{10}$. Na paciente descrita no relato de caso houve uma ótima resposta com o uso da medicação.

\section{CONCLUSÃO}

O insulinoma é um tumor neuroendócrino de baixa incidência na população e a principal causa de hiperinsulinismo endógeno. Portanto, diante de episódios de hipoglicemia é uma hipótese diagnóstica a ser levantada. Exames de imagem como USG transabdominal, tomografia computadorizada e RNM podem ser realizados. Dosagem de glicemia sérica, peptídeo $\mathrm{C}$, proinsulina e sulfoniluréia durante um episódio de hipoglicemia após jejum prolongado ajudam a corroborar a hipótese de insulinoma. No caso descrito acima, foram realizados exames de imagem, porém sem evidência do tumor. Foi encaminhada para abordagem cirúrgica com ressecção do tumor pancreático benigno menor que $2 \mathrm{~cm}$ evidenciado pelo laudo anatomopatológico. Pela impossibilidade de controle da doença e cura pela cirurgia foi optado por utilização de análogo da

Tabela 2. Sintomas e valores de glicose, insulina e peptídeo $\mathrm{C}$ séricos para o diagnóstico de insulinoma e seus diagnósticos diferenciais ${ }^{1}$.

\begin{tabular}{|c|c|c|c|c|}
\hline Diagnóstico & Sintomas & $\begin{array}{c}\text { Glicose } \\
(\mathrm{mg} / \mathrm{dL})\end{array}$ & $\begin{array}{c}\text { Insulina } \\
(\mu \mathrm{U} / \mathrm{mL})\end{array}$ & $\begin{array}{c}\text { Peptídeo C } \\
(\mathrm{nmol} / \mathrm{L})\end{array}$ \\
\hline Normal & Não & $>45$ & $\geq 3$ & $<0,2$ \\
\hline Insulina exógena & Sim & $\leq 45$ & $\geq 3$ a 5 & $\geq 0,2$ \\
\hline Insulinoma & Sim & $\leq 45$ & $\geq 5$ & $\geq 0,2$ \\
\hline Autoinduzida por Sulfoniuréia & Sim & $\leq 45$ & $<5$ & $<$ \\
\hline
\end{tabular}


somatostatina com sucesso. Ressaltamos a dificuldade no diagnóstico da paciente mesmo diante de sintomas neuroglicopênicos clássicos levando a atraso diagnóstico. É essencial aventar o diagnóstico em casos de crise convulsiva que não respondam ao tratamento clássico com anticonvulsivantes e com sintomas glicopênicos.

\section{REFERÊNCIAS}

1. Vilar, L. et al; Endocrinologia Clínica. Sexta edição. Rio de Janeiro: Guanabara Koogan, 2016.

2. Mathur, A.; Gorden, P.; Libutti, S. Insulinoma. Surgical Clinics of North America, v. 89, n. 5, p. 1105-1121, 2009.

3. Okabayashi, T. Diagnosis and management of insulinoma. World Journal of Gastroenterology, v. 19, n. 6, p. 829, 2013.

4. Bhalla S, Punia VPS, Narang M, Kumari P, Gupta S. Seizures due to insulinoma - a rare but treatable cause. Journal of the association of Physicians of India. 2017; 65: 104-105.

5. Shin, J Gorden, P,; Libutti, S. Insulinoma: pathophysiology, localization and management. Future Oncology, v. 6, n. 2, p. 229-237, 2010.

6. Whipple AO, Frantz VK. Adenoma of islet cells with hyperinsulinism: a review. Ann Surg. 1935; 101:1299-1335.

7. Metz DC, Jensen RT. Gastrointestinal neuroendocrine tumors: pancreatic endocrine tumors. Gastroenterology. 2008; 135:1469-1492.

8. Finlayson $\mathrm{E}$, Clark $\mathrm{OH}$. Surgical treatment of insulinomas. Surg Clin N Am. 2004; 84:775-785.

9. Boukhman MP, Karam JH, Shaver J, Siperstein AE, Duh QY, Clark OH. Insulinoma - experience from 1950 to 1995. West J Med. 1998; 169:98-104.

10. Vezzosi D, Bennet A, Rochaix P, et al. Octreotide in insulinoma patients: efficacy on hypoglycemia, relationships with octreoscan scintigraphy and immunostaining with anti-sst2 and anti-sst5 antibodies. Eur J Endocrinol. 2005; 152:757-767.

11. Bhalla S, Punia VPS, Narang M, Kumari P, Gupta S. Seizures due to insulinoma - a rare but treatable cause. Journal of the association of Physicians of India. 2017; 65: 104-105. 